\title{
Origin and Magnitude of 'Designer' Spin-Orbit Interaction in Graphene on Semiconducting Transition Metal Dichalcogenides
}

\author{
Zhe Wang, ${ }^{1, *}$ Dong-Keun Ki, ${ }^{1}$ Jun Yong Khoo, ${ }^{2}$ Diego Mauro, ${ }^{1}$ Helmuth Berger, ${ }^{3}$ \\ Leonid S. Levitov, ${ }^{2}$ and Alberto F. Morpurgo ${ }^{1, t}$ \\ ${ }^{1}$ Department of Quantum Matter Physics (DQMP) and Group of Applied Physics (GAP), \\ University of Geneva, 24 quai Ernest-Ansermet, CH-1211 Genève 4, Switzerland \\ ${ }^{2}$ Department of Physics, Massachusetts Institute of Technology, Cambridge, Massachusetts 02139, USA \\ ${ }^{3}$ Institut de Physique de la Matière Complexe, Ecole Polytechnique Federale de Lausanne, \\ CH-1015 Lausanne, Switzerland
}

(Received 30 May 2016; revised manuscript received 27 July 2016; published 26 October 2016)

\begin{abstract}
We use a combination of experimental techniques to demonstrate a general occurrence of spin-orbit interaction (SOI) in graphene on transition metal dichalcogenide (TMD) substrates. Our measurements indicate that SOI is ultrastrong and extremely robust, despite it being merely interfacially induced, with neither graphene nor the TMD substrates changing their structure. This is found to be the case irrespective of the TMD material used, of the transport regime, of the carrier type in the graphene band, or of the thickness of the graphene multilayer. Specifically, we perform weak antilocalization (WAL) measurements as the simplest and most general diagnostic of SOI, and we show that the spin relaxation time is very short (approximately $0.2 \mathrm{ps}$ or less) in all cases regardless of the elastic scattering time, whose value varies over nearly 2 orders of magnitude. Such a short spin-relaxation time strongly suggests that the SOI originates from a modification of graphene band structure. We confirmed this expectation by measuring a gatedependent beating, and a corresponding frequency splitting, in the low-field Shubnikov-de Haas magnetoresistance oscillations in high-quality bilayer graphene devices on $\mathrm{WSe}_{2}$. These measurements provide an unambiguous diagnostic of a SOI-induced splitting in the electronic band structure, and their analysis allows us to determine the SOI coupling constants for the Rashba term and the so-called spinvalley coupling term, i.e., the terms that were recently predicted theoretically for interface-induced SOI in graphene. The magnitude of the SOI splitting is found to be on the order of $10 \mathrm{meV}$, more than 100 times greater than the SOI intrinsic to graphene. Both the band character of the interfacially induced SOI and its robustness and large magnitude make graphene-on-TMD a promising system to realize and explore a variety of spin-dependent transport phenomena, such as, in particular, spin-Hall and valley-Hall topological insulating states.
\end{abstract}

DOI: 10.1103/PhysRevX.6.041020

\section{INTRODUCTION}

Recently, van der Waals (vdW) heterostructures formed by vertical stacks of different two-dimensional (2D) materials have emerged as designer systems, providing a new paradigm for engineering novel electronic media with widely tunable parameters [1]. Stacked vdW heterostructures nicely combine the ability to tailor interfacial interactions at the atomic scale, while at the same time preserving the integrity

\footnotetext{
*Corresponding author. zhe.wang@unige.ch

Corresponding author Alberto.Morpurgo@unige.ch

Published by the American Physical Society under the terms of the Creative Commons Attribution 3.0 License. Further distribution of this work must maintain attribution to the author(s) and the published article's title, journal citation, and DOI.
}

Subject Areas: Condensed Matter Physics, Graphene, Materials Science of individual layers. This "designer" approach is epitomized by recent work on graphene paired with hexagonal boronnitride ( $\mathrm{hBN}$ ) [2-4]. In this system, a dramatic change in the graphene band structure occurs when the crystal axes of graphene and hBN layers are nearly aligned, in the total absence of any reorganization of chemical bonding or any change in the atomic order of individual layers. The transformed band structure manifests itself in striking and robust transport phenomena-such as the appearance of so-called satellite Dirac points - that are readily observable experimentally. These unexpected findings are opening up a wide avenue of research exploring vdW heterostructures based on many different 2D materials [5-10]. A key goal at this stage is to identify the interfacial interactions that can alter specific electronic properties of interest and to understand the microscopic physical processes responsible for their origin.

One fascinating question in this vein is whether vdW heterostructures can be used to control not only the orbital 
dynamics of electrons in graphene but also their spin, i.e., whether vdW heterostructures can be employed to generate a strong spin-orbit interaction (SOI) in the graphene Dirac band. To this end, combining graphene with large-gap semiconducting transition metal dichalcogenides (TMDs) appears to be a promising route [11] because semiconducting TMDs exhibit an extremely strong SOI [12-15] and because they are known to preserve the high electronic quality of graphene when used as substrates [16]. Recent magnetotransport measurements performed on grapheneon- $\mathrm{WS}_{2}[17-19]$-and, in particular, the observation of a pronounced weak antilocalization (WAL) contribution to the conductivity of graphene [18,19]—confirm these expectations. Indeed, the analysis of the experimental results indicated that the spin relaxation time $\tau_{\text {so }}$ in graphene-on- $\mathrm{WS}_{2}$ is between 100 and 1000 times shorter than in graphene on $\mathrm{SiO}_{2}$ [20] or on hBN [21]. This is broadly consistent with ab initio calculations, which predict the strength of SOI in graphene-on-WS $\mathrm{WS}_{2}$ to be at least 100 times larger than the SOI intrinsic to graphene [18,22-24].

These results pose a number of interesting and challenging questions, which are central for our understanding of the new phenomenon of designer SOI. In particular, the physical process by which strong SOI can be imprinted by one layer on an adjacent layer without any changes in their structure remains puzzling. So far, the experiments have been unable to elucidate the microscopic mechanism responsible for the strong SOI in graphene, nor have they provided any reliable insight into the functional form of the induced SOI or its strength. In addition, they did not establish whether a strong interfacially induced SOI is unique to graphene-on- $\mathrm{WS}_{2}$ or whether it is a robust, generic property of all graphene-on-TMD heterostructures. Last but not least, perhaps the most tantalizing question of all is whether SOI is dominated by disorder scattering or by a band structure modification. If the latter happens to be the case, the strong SOI present in a graphene-on-TMD system can be employed to create and explore a variety of electronic media with novel properties [25-27].

Here, we exploit a variety of graphene-on-TMD heterostructures to tackle these questions in a comprehensive way. A large part of the work focuses on the study of WAL in heterostructures formed by graphene and one of the semiconducting TMDs: $\mathrm{WSe}_{2}, \mathrm{MoS}_{2}$, and $\mathrm{WS}_{2}$. For all TMDs used, irrespective of carrier mobility $(\mu)$, position of the Fermi level in graphene, and thickness of the graphene layer (up to trilayer), a pronounced WAL signal is observed. This finding shows that interfacially induced SOI in graphene-on-TMDs is an extremely robust phenomenon, insensitive to virtually all details of the $\mathrm{vdW}$ heterostructure considered. A quantitative analysis of the WAL data allows us to establish an upper bound of approximately $0.2 \mathrm{ps}$ for the spin-relaxation time $\tau_{\text {so }}$, irrespective of the carrier mobility (which was varied by nearly 2 orders of magnitude). Such a short $\tau_{\text {so }}$ value appears to be physically compatible only with SOI originating from a modification of the graphene band structure. To validate this conclusion, we present measurements of Shubnikov-de Haas $(\mathrm{SdH})$ conductance oscillations exhibiting a beating due to a splitting in their frequency [28-30]. The size of the splitting and its dependence on carrier density show that the beating originates from SOI, and its quantitative analysis allows us to establish the SOI magnitude. We find that the dominant SOI term is of the Rashba type and that its characteristic energy is approximately $\lambda_{R} \simeq 10-15 \mathrm{meV}$; the strength of the other SOI term expected to be induced by interfacial interactions [18]-i.e., the one that couples spin and valley—ranges between $\lambda=0$ and $\lambda \approx 5-6 \mathrm{meV}$ (i.e., experimental data are compatible with $\lambda_{R}=15 \mathrm{meV}$ and $\lambda=0 \mathrm{meV}$ or with $\lambda_{R}=10 \mathrm{meV}$ and $\lambda=5-6 \mathrm{meV}$, as well as different choices in these intervals).

Besides elucidating most aspects of interfacially induced SOI in graphene, the results presented here clearly illustrate the experimental flexibility of graphene-on-TMDs heterostructures. These heterostructures allow comparative studies by varying the specific TMD material used, the thickness of the graphene layer, the position of the Fermi level, and the scattering time $(\tau)$. Even more flexibility could be introduced using double gated devices, to tune the graphene band structure (e.g., in bilayers) [31-34] and further extend the range of carrier densities accessible experimentally. We anticipate that this unrivaled experimental flexibility will prove useful in future experiments aiming at exploring other aspects of interfacial interactions in $\mathrm{vdW}$ heterostructures.

\section{DEVICE FABRICATION AND CHARACTERIZATION}

The van der Waals heterostructures were assembled by transferring graphene layers of different thickness (monolayers, bilayers, or trilayers) onto thin exfoliated flakes of TMDs $\left(\mathrm{WSe}_{2}, \mathrm{WS}_{2}\right.$, and $\mathrm{MoS}_{2}$ ), resulting in devices whose cross section is schematically shown in Fig. 1(a). For the assembly, we employed a commonly used drytransfer technique [35,36]. Conventional electron-beam lithography, liftoff, and oxygen plasma etching techniques were employed to pattern and contact multiterminal Hall-bar devices. The contacts consisted of an evaporated $\mathrm{Ti} / \mathrm{Au}$ thin film $(10 / 70 \mathrm{~nm})$. All structures were realized on substrates consisting of degenerately doped silicon covered with a 285-nm-thick layer of thermally grown $\mathrm{SiO}_{2}$. The charge density $(n)$ of graphene is tuned by operating the doped silicon substrate as a gate electrode. In this configuration, for sufficiently large gate voltage $\left(V_{g}\right)$, carriers are accumulated at the surface of the TMD flake at the interface with the $\mathrm{SiO}_{2}$ layer $[18,37]$. When that happens, the carrier density in graphene cannot be tuned anymore by $V_{g}$, and the conductivity $(\sigma)$ of graphene saturates. 
(a)
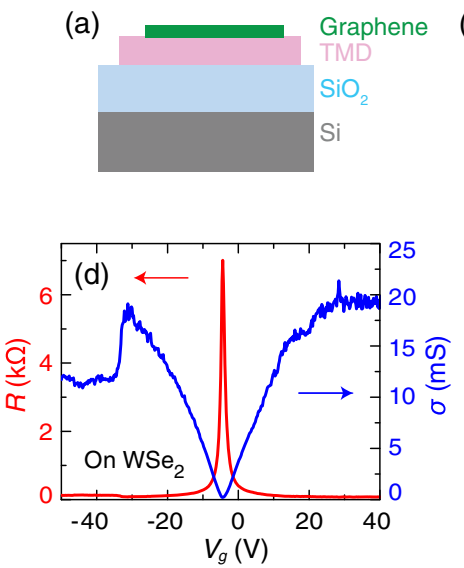

(b)
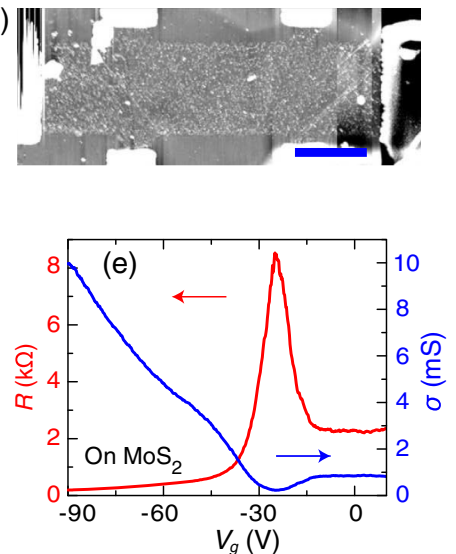

(c)
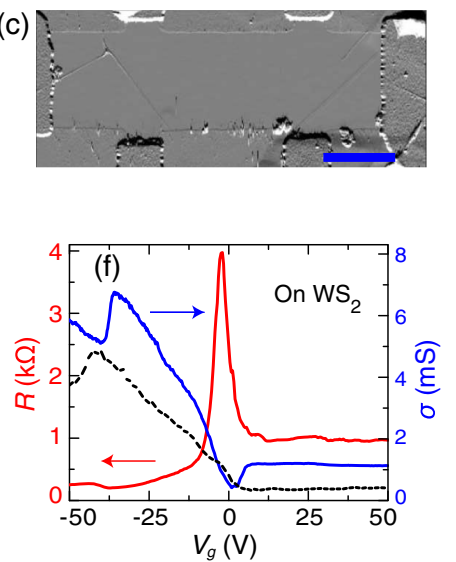

(g)
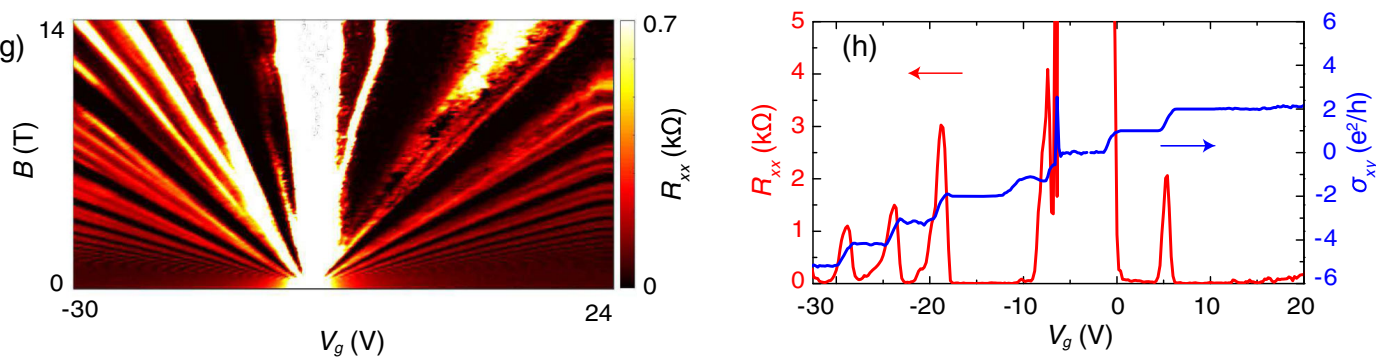

FIG. 1. Basic characterization of graphene on TMD substrates. (a) Schematic cross section of the devices. The graphene layer (green) is transferred onto a TMD crystal (pink) that has previously been exfoliated on a substrate consisting of highly doped silicon (gray) covered by 285 -nm-thick $\mathrm{SiO}_{2}$ (light blue). The silicon substrate is operated as a backgate. (b,c) AFM images of a graphene Hall-bar device before and after the AFM-ironing process (the scale bar is $2 \mu \mathrm{m}$ long). (d-f) Gate-voltage $\left(V_{q}\right)$ dependence of the resistance ( $R$, red curves) and the conductivity ( $\sigma$, blue curves) of monolayer graphene on $\mathrm{WSe}_{2}(\mathrm{~d}), \mathrm{MoS}_{2}(\mathrm{e})$, and $\mathrm{WS}_{2}(\mathrm{f})$, measured at $4.2 \mathrm{~K}$. The carrier mobility in the three cases is 110000,33000 , and $23400 \mathrm{~cm}^{2} / \mathrm{Vs}$, respectively. In all devices, the conductance saturates at large enough $V_{g}$ away from charge neutrality point, when charges start to accumulate at the $\mathrm{SiO}_{2}$-TMD interface. The black line in (f) represents the $V_{g}$-dependent conductivity $\sigma\left(V_{g}\right)$ before AFM ironing (the corresponding mobility is approximately 2 times smaller than for the blue curve). (g,h) Integer quantum-Hall effect (QHE) observed in high-quality graphene-on-WSe $\mathrm{W}_{2}$ at $T=250 \mathrm{mK}$ whose basic characterization is shown in (d). The color map of the longitudinal resistance $\left(R_{x x}\right)$ versus $V_{g}$ and $B(\mathrm{~g})$ and the $V_{g}$ dependence of $R_{x x}$ (red curves) and the Hall conductance ( $\sigma_{x y}$, blue curves) measured at $B=12 \mathrm{~T}$ (h) clearly confirm the occurrence of the vanishing $R_{x x}$ and concomitantly quantized $\sigma_{x y}=\nu \times e^{2} / h$ at integer values of filling factor $(\nu \equiv n h / e B)$. In panel (h), the QH plateaus at $\nu= \pm 1$, $-3,-4,-5$ due to the full degeneracy lifting of the $N=0,1$ Landau levels in monolayer are clearly visible.

The device quality-i.e., the carrier mobility and the inhomogeneity in carrier density-depends on the details of the assembly process and on the procedure to "clean" the device structure at the end of the fabrication. Two key elements introduce disorder: structural defects resulting from the transfer process, such as "bubbles" and "wrinkles" in the graphene layer [38], and adsorbates adhering onto graphene (mostly polymer residue remaining at the end of the fabrication process). The influence of both elements can be controlled in different ways. Structural defects can be eliminated by defining the graphene Hall bar in regions in which these defects are absent. Selecting these areas, which typically have linear dimensions of the order of 5-6 $\mu \mathrm{m}$, usually results in very high carrier mobility: We have observed low-temperature mobility values as large as $160000 \mathrm{~cm}^{2} / \mathrm{Vs}$, comparable to (or possibly even slightly better than) the best values observed in graphene-on-hBN structures assembled by the same dry-transfer technique
$[2-4,39,40]$. Selecting larger areas is also possible, but this unavoidably prevents the full exclusion of structural defects, resulting in lower mobility.

Adsorbates can be eliminated in a rather controlled way by a so-called atomic force microscope (AFM) "ironing" process [41], which is essential to realize high-mobility devices. AFM ironing consists of scanning the graphene flake with an AFM tip in contact mode, applying only a moderate force, in such a way as to pile up all the adsorbates just outside the edges of the graphene flake. The effectiveness of the process is illustrated in Figs. 1(b) and 1(c). Figure 1(b) shows an AFM image of a device at the end of fabrication, and Fig. 1(c) shows another image of the same device taken after the ironing step. The difference-the extremely small corrugation that is measured on graphene after the ironing process - is clear. In our studies, we have measured 16 different devices, in whichdepending on their area, cleaning procedure adopted, 
density of bubbles, etc.- the carrier mobility extracted from measurements of the conductivity and of the Hall effect ranged between 3000 and $160000 \mathrm{~cm}^{2} / \mathrm{Vs}$.

Figures 1(d)-1(f) show the gate-voltage dependence of the resistance (red curves) and of the corresponding conductivity (blue curves) measured on three representative devices, respectively- $\mathrm{WSe}_{2}, \mathrm{MoS}_{2}$, and $\mathrm{WS}_{2}$. As compared to our earlier work on graphene-on- $\mathrm{WS}_{2}$, in which no AFM ironing was done [18], in the current generation of higher-quality devices, the charge neutrality point is "exposed" in all cases: It is possible to shift the Fermi level $\left(E_{F}\right)$ both in the valence and in the conduction band by acting on the backgate. Whereas for graphene on $\mathrm{WS}_{2}$ and $\mathrm{MoS}_{2}$ only a small range of energies in the conduction band can be accessed, for $\mathrm{WSe}_{2}, E_{F}$ can be shifted over a rather large interval in both the valence and the conduction band. Hence, $\mathrm{WSe}_{2}$ allows the systematic investigation of SOI in the conduction band without the need to use a top gate electrode, something that could not be done in previous work. Finally, Figs. 1(g) and 1(h) show that all integer QHE states are visible, including the symmetry-broken states caused by the presence of electron-electron interactions [39], which is indicative of the high quality of the devices (in the best cases, symmetry-broken states become visible already for applied magnetic fields as low as approximately 1 Tesla).

\section{EXTRACTING SOI FROM WEAK ANTILOCALIZATION IN MONOLAYER GRAPHENE ON DIFFERENT TMDS}

Weak antilocalization is a striking quantum interference effect originating from spin-orbit coupling that has long served as a direct probe of SOI in conductors $[42,43]$. Also for graphene-on-TMD heterostructures, the observation of the WAL correction to the low-temperature magnetoconductivity provides the simplest and most general diagnostic of the presence of SOI [18]. Extracting the WAL contribution requires suppressing the effect of the so-called phasecoherent universal conductance fluctuations (UCF) originating from random interference of electronic waves [44]. Indeed, since the dimensions of our graphene-on-TMD devices are typically comparable to (or even smaller than) the phase coherence length $L_{\phi}$, the WAL contribution in any individual measurement is normally eclipsed by the presence of UCF. To make the WAL contribution stand out, we suppress the magnitude of the UCF by looking at the ensemble-averaged conductivity, obtained by averaging many (typically 50) magnetoconductance traces measured at slightly different values of $V_{g}$ (the procedure is identical to that described in Ref. [18] to which we refer for details).

The procedure described above, performed at different temperatures, leads to the results shown in Figs. 2(a)-2(c) for graphene on $\mathrm{WSe}_{2}, \mathrm{MoS}_{2}$, and $\mathrm{WS}_{2}$ respectively. In all cases, a negative magnetoconductivity of order $e^{2} / h$ is
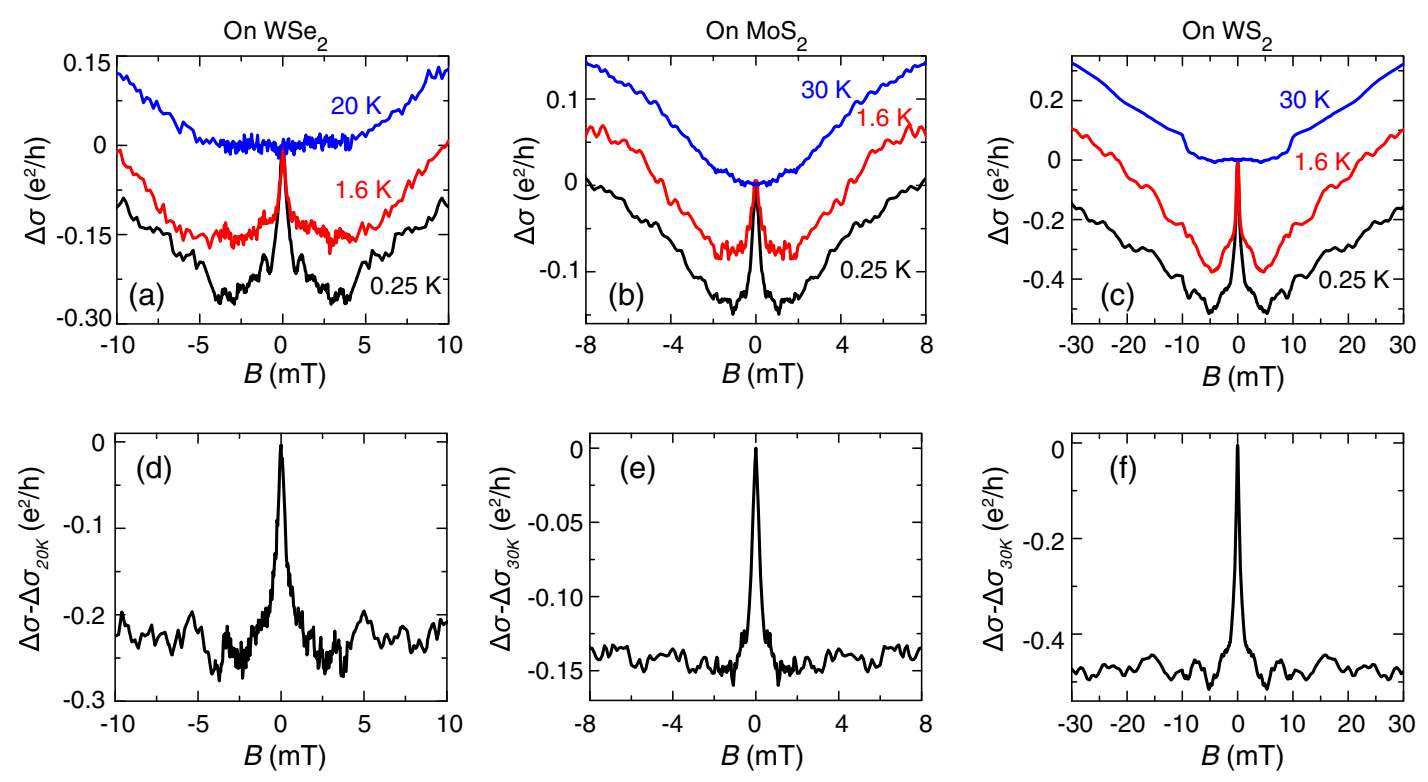

FIG. 2. Negative magnetoconductivity due to WAL in monolayer graphene on TMD substrates. (a-c) Ensemble-averaged magnetoconductivity measured on monolayer graphene on $\mathrm{WSe}_{2}, \mathrm{MoS}_{2}$, and $\mathrm{WS}_{2}$, for $T=250 \mathrm{mK}$ (black curve), $T=1.6 \mathrm{~K}$ (red curves), and $T=20$ or $30 \mathrm{~K}$ (blue curves), with the Fermi level gate-tuned to be in the graphene valence band. The data are measured on the same devices whose $V_{g}$-dependent transport curves are shown in Figs. 1(d)-1(f). The characteristic peak due to WAL around $B=0 \mathrm{~T}$ is clearly visible at low temperatures and disappears at higher temperatures. (d-f) Quantum corrections to magnetoconductivity of monolayer graphene on $\mathrm{WSe}_{2}, \mathrm{MoS}_{2}$, and $\mathrm{WS}_{2}$, obtained by subtracting the classical contribution (corresponding to the magnetoconductivity measured at $T=20$ or $30 \mathrm{~K}$ ) from the magnetoconductivity measured at $T=250 \mathrm{mK}$. Note that up to the highest magnetic field investigated, no signatures of weak localization are visible. 
clearly apparent at the lowest temperature investigated, $T=250 \mathrm{mK}$, upon the application of a magnetic field $(B)$ of a few milliTesla. The magnitude of the negative magnetoconductivity decreases upon warming up the devices, and the effect disappears entirely at $T \simeq 20-30 \mathrm{~K}$, as expected for quantum interference effects [43]. We conclude that, irrespective of the TMD used to realize the heterostructures, the presence of a pronounced WAL signal in magnetotransport demonstrates that in all cases SOI is induced in graphene.

In extracting the strength of SOI from magnetotransport measurements, special care should be taken into accounting for the interplay between WAL and weak localization since the two effects contribute to magnetoconductivity with opposite signs [45]. Weak localization gives rise to a positive magnetoconductivity, which becomes visible when, during the phase-coherent propagation of electrons, their spins do not rotate, or rotate by a small enough angle [42]. Despite the presence of SOI, a small positive magnetoconductivity due to weak localization may still be observed because, at sufficiently high magnetic fields, only the shortest trajectories give a non-negligible contribution to the interference effects probed by the ensembleaveraged conductivity. Unless SOI is extremely strong, the electron spin may not have time to rotate by a sufficient amount along these short trajectories, and signatures of weak localization may then become visible at large $B$.

The issue is relevant because-as it is clear from Figs. 2(a)-2(c) - a positive magnetoconductivity is visible in the measurements, and it is important to establish whether this is a manifestation of weak localization. To this end, we recall that WAL and weak localization are quantum corrections to the conductivity; i.e., they correspond to the difference between the total magnetoconductivity that is actually measured and the classical contribution. The classical contribution is straightforward to determine since it corresponds to the magnetoconductivity measured at sufficiently high temperatures, where phase-coherent effects have been suppressed because of the thermally induced shortening of $L_{\phi}$. Since at 20-30 K the effect of WAL has entirely disappeared and the remaining low-field (positive) magnetoconductivity does not exhibit any significant temperature dependence, we can take the magnetoconductivity measured at these temperatures to be a good approximation of the classical contribution (see the Appendix for more details on the classical magnetoconductivity background). The resulting quantum correction to the magnetoconductivity for the different devices is shown in Figs. 2(d)-2(f). Within the precision of the measurements, determined by the remnant amplitude of UCF fluctuations, no positive magnetoconductivity is visible in Figs. 2(d)-2(f). We conclude that irrespective of the TMD material used in the heterostructure, a clear WAL signal is always present with no detectable weak-localization contribution. This observation provides a first clear indication that in all heterostructures investigated, the SOI induced in graphene has a very strong intensity.

Data measured on other monolayer graphene devices confirm that WAL always occurs with no detectable weaklocalization signal, irrespective of whether carriers are electrons or holes, and of their mobility (or, equivalently, scattering time $\tau$ ), which we varied over a range of nearly 2 orders of magnitude. Neither aspect had been addressed in our previous work on $\mathrm{WS}_{2}$, in which the Fermi level could not be shifted into the conduction band and the scattering time was only varied by a limited amount [18]. The occurrence of WAL for electron transport is best illustrated with data measured on graphene-on- $\mathrm{WSe}_{2}$, shown in Fig. 3(a), in which a fully developed WAL signal is clearly visible. The effect of the mobility can be appreciated by looking at Figs. 3(b) and 3(c). Figure 3(b) shows the quantum correction to the magnetoconductivity measured on a large-area graphene-on- $\mathrm{WSe}_{2}$ device, in which a high density of bubbles led to a low-temperature mobility of $3000 \mathrm{~cm}^{2} / \mathrm{Vs}$ [whereas all devices shown in Figs. 2(a)-2(c) had mobility larger than $25000 \mathrm{~cm}^{2} / \mathrm{Vs}$ ]. We find that the magnitude of the WAL correction is comparable in all cases, but in the lower mobility devices, the magnetic field required to observe the negative magnetoconductivity is larger. This result is expected since when the mobility is lower, a larger magnetic field is needed to pierce a flux of $\Phi_{0}=h / e$ through the area in
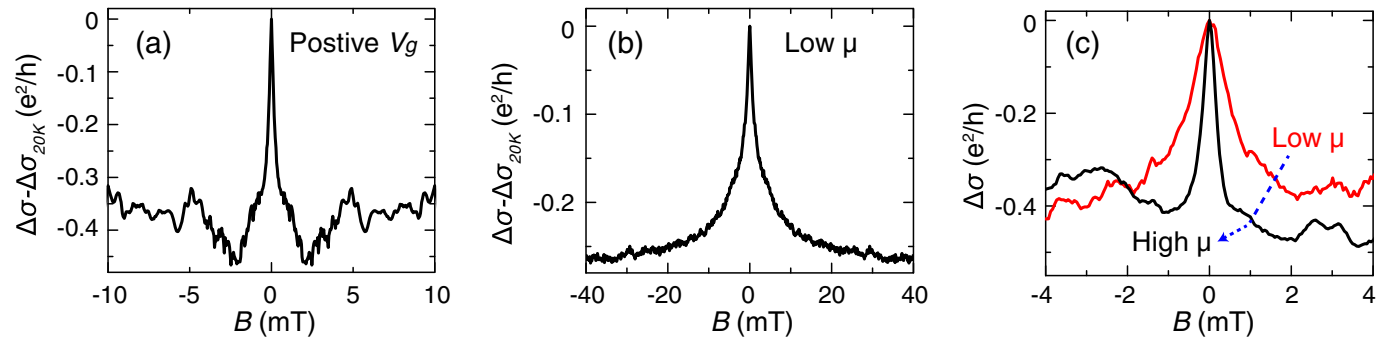

FIG. 3. Interfacially induced SOI in graphene-on-TMD is a robust phenomenon. (a) Magnetoconductivity of graphene-on-WSe 2 measured with the Fermi energy gate-tuned to be in the graphene conduction band. (b) Magnetoconductivity due to WAL measured on a larger-area graphene-on-WSe ${ }_{2}$ device with a carrier mobility of only $3000 \mathrm{~cm}^{2} / \mathrm{Vs}$. (c) Magnetoconductivity of graphene-on-WS measured on the same device before (red) and after (black) the AFM-ironing process needed to clear graphene from adsorbates. As the carrier mobility increases, the WAL peak becomes sharper, as expected. All data in this figure have been measured at $T=250 \mathrm{mK}$. 
which the electronic waves propagate phase coherently and interfere. The same conclusions can be drawn by looking at Fig. 3(c), which shows the WAL magnetoconductivity in a same graphene-on- $\mathrm{WS}_{2}$ device measured before (red curve) and after (black curve) performing an AFM ironing step, resulting in a mobility increase. It is apparent that, in this case as well, a higher mobility leads to a decrease of the magnetic field scale needed to suppress the effect of WAL.

\section{SOI IN BILAYER GRAPHENE AND THICKER MULTILAYERS}

When monolayer graphene is substituted by thicker multilayers, a strong SOI remains present in all devices (irrespective of the TMD used for the device realization). Figure 4 illustrates this conclusion with data measured on two different graphene-on-WSe ${ }_{2}$ devices: Figures 4(a)-4(c) refer to a bilayer graphene device with low-temperature mobility $\mu=33000 \mathrm{~cm}^{2} / \mathrm{Vs}$ and Figs. 4(d)-4(f) to a trilayer having mobility $\mu \simeq 110000 \mathrm{~cm}^{2} /$ Vs. Basic transport characterization shows that-as for monolayer devices-heterostructures based on thicker multilayers exhibit an excellent electronic quality. The resistance peak around the charge neutrality point is extremely sharp in both cases [see Figs. 4(a) and 4(d)]; in the trilayer device, the measured width corresponds to a charge inhomogeneity as low as $1.8 \times 10^{10} \mathrm{~cm}^{-2}$, comparable to the best-reported width for nonsuspended graphene devices. In the presence of a perpendicular magnetic field $B=4 \mathrm{~T}$, the expected Hall effect quantization sequence is observed in the bilayer device, with plateaus in the Hall conductance occurring at $\sigma_{x y}=4 N e^{2} / h(N= \pm 1, \pm 2, \ldots)[46,47]$, concomitantly with the vanishing of the longitudinal resistance. In the thicker multilayer, the plot of the longitudinal resistance versus filling factor $\nu \equiv n h / e B$ and $B$ [Fig. 4(e)] shows the appearance of broken-symmetry quantum Hall states already at $B$ as low as approximately $1 \mathrm{~T}$. In short, excellent quality bilayer and trilayer graphene devices can be realized on TMD substrates, comparable to the very best devices realized on $\mathrm{hBN}$ by means of the same technique.

Figures 4(c) and 4(f) show that a pronounced lowtemperature negative magnetoconductivity due to WAL is clearly visible in both the bilayer and the trilayer device. In these devices as well, no background due to weak localization is observed, indicative of the large SOI strength. This is remarkable because interfacial interactions are expected to modify only the properties of the bottom graphene layer, the one in direct contact with the TMD crystal [18]. Under normal conditions, the eigenfunctions in the different bands of the multilayer are such that
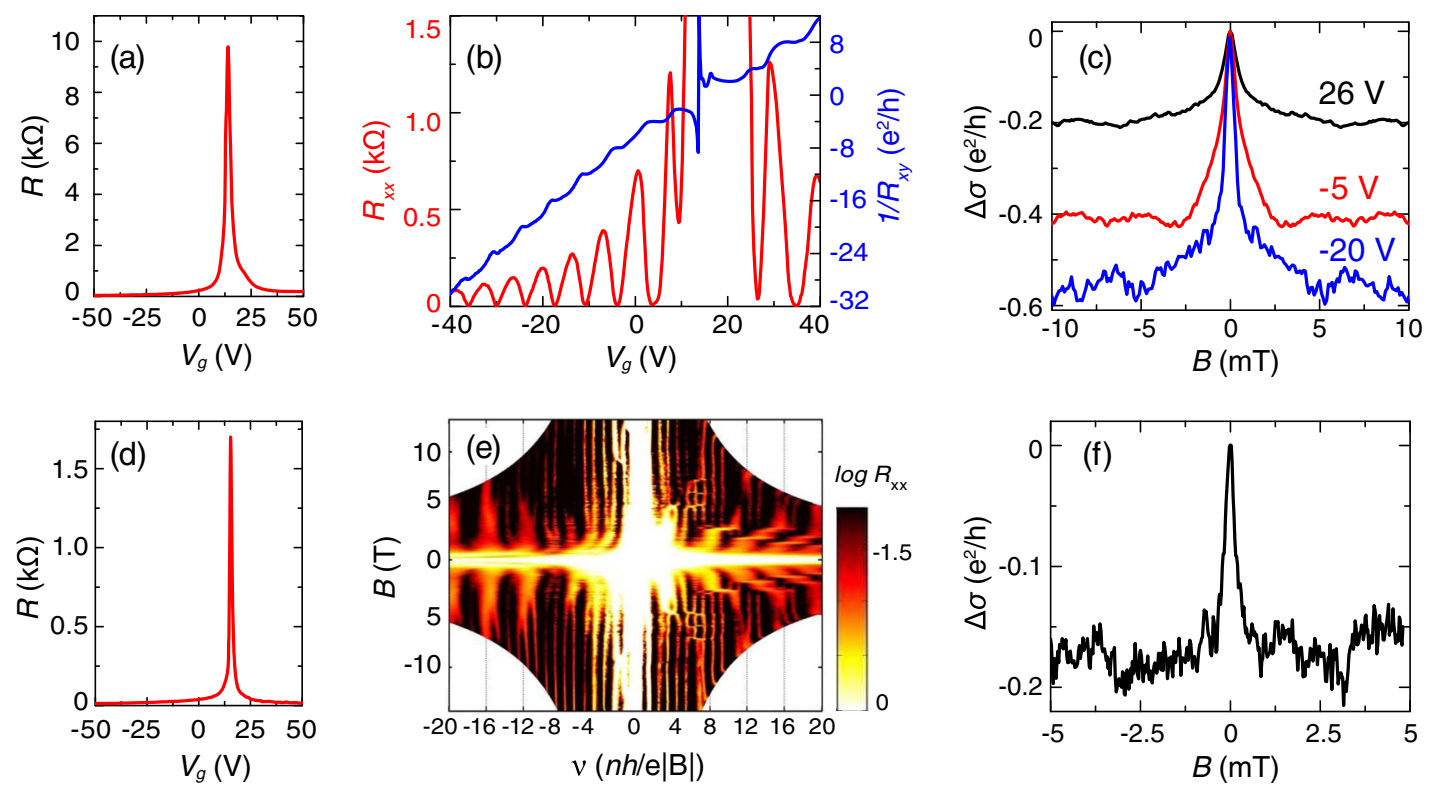

FIG. 4. Interfacially induced SOI in graphene multilayers on TMDs. Panels $(\mathrm{a}-\mathrm{c})$ and $(\mathrm{d}-\mathrm{f})$ refer, respectively, to a bilayer and a trilayer device, both realized on $\mathrm{WSe}_{2}$. Panels (a) and (d) show that the resistance peak around charge neutrality is very narrow, indicative of a high uniformity of carrier density (in the trilayer, the magnitude of inhomogeneity is smaller than $2 \times 10^{10} \mathrm{~cm}^{-2}$, comparable to the best devices on hBN). A high-quality integer quantum Hall effect is observed in both devices, as shown by data in panels (b) and (e) measured at $T=4.2 \mathrm{~K}$. In panel (b), in which the characteristic quantization sequence of graphene bilayer is observed, the applied magnetic field is $B=4 \mathrm{~T}$. Panel (e) shows a color plot of the longitudinal resistance of trilayer graphene as a function of filling factor and magnetic field. Full breaking of the degeneracy of Landau levels due to electron-electron interactions starts to be clearly visible already at $B=1 \mathrm{~T}$. Panels (c) and (f) show the ensemble-averaged magnetoconductivity measured at $T=250 \mathrm{mK}$, exhibiting the characteristic signature of WAL, with no positive magnetoconductivity due to weak localization [in panel (c), the different curves show data at different gate voltages, corresponding to shifting the Fermi level from the bilayer graphene valence band to the conduction band]. 
electrons have a finite amplitude of probability to be in that layer. As a result, all bands are affected, and that is why thicker multilayers exhibit a pronounced WAL. Nevertheless, it is clear that the effect of interfacially induced SOI should decrease in intensity as the thickness of the multilayer increases since the probability for electrons to enter in contact with the TMD crystal decreases upon increasing thickness (or, equivalently, the amplitude of the electron wave functions in the bottom layer-the one in contact with the TMD-decreases for thicker multilayers). The data shown in Fig. 4(c)—and especially those shown in Fig. 4(f) - indicate that, despite the larger thickness, at least up to trilayer graphene, the observed behavior of the WAL correction is the one typical of very strong SOI. Significantly thicker multilayers are needed to "dilute" the effect of SOI induced by interfacial interactions.

The observation of WAL in graphene bilayers [Fig. 4(c)] is worth an additional comment. In monolayers, WAL can occur because of only the Dirac nature of electrons (i.e., in the absence of SOI), as a consequence of the $\pi$ Berry phase picked up by the electron wave functions that undergo backscattering processes while staying in the same valley. WAL due to this effect has been seen experimentally, albeit only at elevated temperatures (typically $T \geq 10 \mathrm{~K}$ ) [48] since only then is $L_{\phi}$ sufficiently short (such a dependence on temperature allows the phenomenon to be discriminated from WAL due to SOI, which increases in amplitude upon cooling). The effect is absent in bilayer graphene since in bilayers a $2 \pi$ Berry phase is acquired by the electron wave function upon backscattering, which does not lead to WAL [49]. As such, the occurrence of WAL in bilayers illustrated by the data shown in Fig. 4(c) provides unambiguous and more direct evidence of the presence of interfacially induced SOI.

In concluding this section, we emphasize that the possibility of using interfacial interactions with a TMD substrate to induce strong SOI in different graphene multilayers - and not only in monolayers-adds to the flexibility of this experimental system. As we will discuss in Sec. VI, we exploit this flexibility in the present work to quantitatively determine the type and magnitude of the interfacially induced SOI.

\section{QUANTITATIVE ANALYSIS OF THE WEAK ANTILOCALIZATION DATA}

The pronounced WAL observed in the measurements presented in the previous sections, together with the absence of any signature of weak localization, illustrates that strong SOI is a general property of graphene-TMD heterostructures. For a quantitative analysis, we confine ourselves to devices realized on monolayers, for which an explicit theoretical expression for the magnetoconductivity due to WAL is available [50]. The expression, which takes into account the effect of all possible symmetry-allowed SOI terms, reads

$$
\begin{aligned}
\Delta \sigma(B)= & -\frac{e^{2}}{\pi h}\left[F\left(\frac{\tau_{B}^{-1}}{\tau_{\phi}^{-1}}\right)-F\left(\frac{\tau_{B}^{-1}}{\tau_{\phi}^{-1}+2 \tau_{\mathrm{asy}}^{-1}}\right)\right. \\
& \left.-2 F\left(\frac{\tau_{B}^{-1}}{\tau_{\phi}^{-1}+\tau_{\mathrm{so}}^{-1}}\right)\right],
\end{aligned}
$$

where $F(x)=\ln (x)+\psi(1 / 2+1 / x)$, with $\psi(x)$ the digamma function. Here, $\tau_{B}^{-1}=4 D e B / \hbar$ ( $D$ is the carrier diffusion constant), $\tau_{\phi}^{-1}$ is the dephasing rate, $\tau_{\text {asy }}^{-1}$ represents the spin relaxation rate due to the SOI terms that break $z \rightarrow-z$ symmetry ( $z$ is the direction normal to the graphene plane), and $\tau_{\mathrm{so}}^{-1}$ is the total spin-relaxation rate, including the effect of all SOI terms.

Since weak (anti)localization theory is developed having the fully diffusive transport regime in mind [50], we start with the analysis of the magnetoconductivity measured in the lowest mobility device, i.e., the graphene-on- $\mathrm{WSe}_{2}$ device whose data are shown in Fig. 3(b) (carrier mobility $\mu \cong 3000 \mathrm{~cm}^{2} / \mathrm{Vs}$; elastic scattering time $\tau \approx 0.04 \mathrm{ps}$ ). Figure 5(a) shows that Eq. (1) reproduces the data well

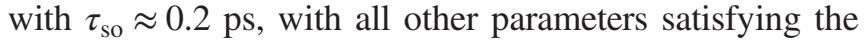
conditions of validity of the theory: $\tau_{\phi}>\tau_{\text {asy }}>\tau_{\text {so }}>\tau$ [51]. However, more considerations are needed to
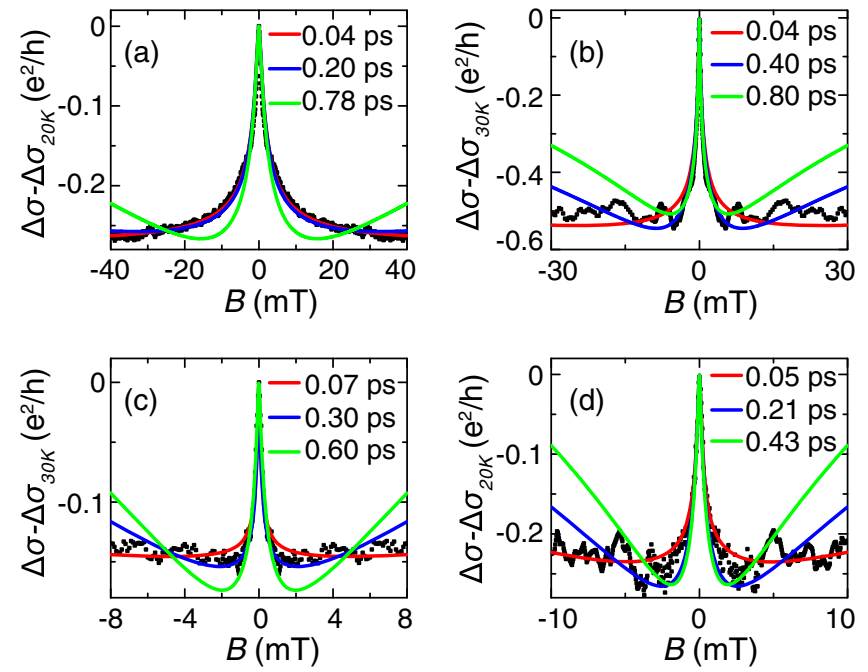

FIG. 5. Comparison of WAL data measured on monolayer graphene on different TMDs with the theoretical predictions of Eq. (1). In panels (a-d), the black dots represent the data; the solid lines of different colors represent the predictions of Eq. (1) for different values of spin-relaxation time $\tau_{\text {so }}$. In panel (a), data from a low-mobility device $\left(\mu=3000 \mathrm{~cm}^{2} / \mathrm{Vs}\right)$ on $\mathrm{WSe}_{2}$ are shown; panels $(b-d)$ show data on higher mobility devices (respectively, 23 400, 33 000, and $110000 \mathrm{~cm}^{2} / \mathrm{Vs}$ ) on $\mathrm{WS}_{2}$ (b), $\mathrm{MoS}_{2}$ (c), and $\mathrm{WSe}_{2}(\mathrm{~d})$. Note in all cases that inserting values of $\tau_{\mathrm{so}}>0.5 \mathrm{ps}$ in Eq. (1) leads to the appearance of a positive magnetoconductivity due to weak localization at higher $B$, which is not seen in the experiments. This allows us to determine an upper bound for $\tau_{\text {so }}$ for all the devices investigated. All data in this figure have been measured at $T=250 \mathrm{mK}$. 
physically understand the meaning of the good agreement between Eq. (1) and the experimental data.

To this end, we note that, for $\tau_{\text {so }}$ values longer than $0.2 \mathrm{ps}$, theory predicts that weak localization should become visible in the magnetic field range explored in the measurements [see, for instance, the green curve in Fig. 5(a), which represents Eq. (1) with $\tau_{\mathrm{so}}=0.78 \mathrm{ps}$ ], contrary to what is observed experimentally (no positive magnetoconductivity is observed up to the largest magnetic field $B=40 \mathrm{mT}$ applied in the experiments). Therefore, $\tau_{\mathrm{so}}$ values significantly larger than $0.2 \mathrm{ps}$ are not compatible with our observations and can be excluded. However, $\tau_{\text {so }}$ values shorter than $0.2 \mathrm{ps}$ are compatible with the measurements. This is illustrated by the red curve in Fig. 5(a), which is a plot of Eq. (1) with $\tau_{\mathrm{so}}=0.04$ ps (and with all other parameters the same). This choice for $\tau_{\mathrm{so}}$ also perfectly reproduces the data and leads to a magnetoconductivity that is indistinguishable from the case $\tau_{\text {so }}=0.2 \mathrm{ps}$. We therefore conclude that our analysis of WAL can only provide an upper value for $\tau_{\mathrm{so}} \approx 0.2 \mathrm{ps}$.

These considerations make it clear that measuring WAL up to sufficiently high magnetic fields is important because the absence of a positive magnetoconductivity due to weak localization is what allows a more precise quantitative determination of the upper bound for $\tau_{\mathrm{so}}$. In our earlier work on graphene-on-WS $\mathrm{W}_{2}$ [18], in which the analysis of WAL was also used to obtain $\tau_{\text {so }}$, the magnetic-field range had not been extended sufficiently in the measurements. As a result, the estimates of $\tau_{\text {so }}$ reported there are approximately 1 order of magnitude larger than what we find now. In other words, from the analysis reported in our previous work, the intensity of SOI-albeit already very strongappeared to be weaker than what it actually is.

For devices with higher mobility, in which the electron mean-free path $l=v_{F} \tau$ becomes longer, the analysis of WAL requires more critical thinking. For instance, the use of Eq. (1) is meaningful only up to magnetic-field values $B \approx \Phi_{0} / l^{2}$ (where $\Phi_{0}=h / e$ is the quantum of flux) because in the diffusive regime described by Eq. (1), the minimum area of a time-reversed trajectory giving rise to WAL or weak localization is of the order of $l^{2}$ [45]. For devices in which $\mu \simeq 100000 \mathrm{~cm}^{2} / \mathrm{Vs}$, the corresponding value of $B$ is only approximately $5 \mathrm{mT}$. This does not pose problems to observe the characteristic peak in WAL, which for such high $\mu$ values becomes extremely narrow [see, e.g., Fig. 5(c)], but it limits the range of magnetic field that can be meaningfully used in the quantitative analysis of the data. Nevertheless, in practice, we find that Eq. (1) does satisfactorily reproduce the measured magnetoconductivity in all cases, as illustrated in Figs. 5(b) and 5(d). Therefore, we proceed as we discussed for the low mobility case and determine the upper bound for $\tau_{\text {so }}$ for each of the devices analyzed. This upper bound is such that for larger $\tau_{\text {so }}$ values, the predictions of Eq. (1) show the presence of a positive magnetoconductivity at higher magnetic fields and

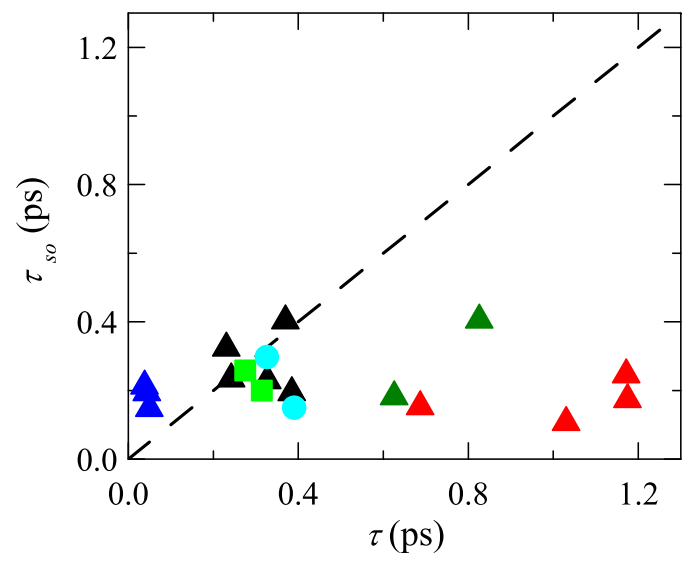

FIG. 6. Upper bound of $\tau_{\text {so }}$, extracted from WAL data, as a function of $\tau$ for monolayer graphene devices. The shape of the different symbols refers to devices realized on different TMD substrates (triangles for $\mathrm{WSe}_{2}$, circles for $\mathrm{MoS}_{2}$, and squares for $\mathrm{WS}_{2}$ ). For each different symbol, data points represented with the same color refer to the same device measured at different gate voltages. The dashed line corresponds to $\tau_{\text {so }}=\tau$.

are incompatible with the experimental observations, whereas for smaller values of $\tau_{\text {so }}$, the magnetoconductivity predicted by Eq. (1) does not change significantly and reproduces the behavior of the experimental data.

The result of this analysis is summarized in Fig. 6, in which the upper bound for $\tau_{\text {so }}$ extracted for all monolayer devices analyzed is plotted as a function of the elastic scattering time $\tau$. For each device, the result of the analysis performed for different applied gate voltage is also shown. The upper bounds for $\tau_{\text {so }}$ determined from WAL cluster between 0.1 and $0.4 \mathrm{ps}$, and in most cases, they are close to $0.2 \mathrm{ps}$. This is a remarkably systematic behavior, especially considering the large range in carrier mobility investigated (from 3000 to $110000 \mathrm{~cm}^{2} / \mathrm{Vs}$ ).

We note that care is needed in interpreting this result because for sufficiently high-mobility devices (e.g., in all cases in which $\tau>0.5$ ps in Fig. 6), $\tau_{\text {so }}<\tau$, which appears to be beyond the regime of validity of Eq. (1) (the assumption that motion is diffusive implies that $\tau$ is the shortest time scale). This situation is not new. It has already been encountered in the analysis of WAL in different twodimensional systems in which SOI is known to be extremely strong, such as 2D hole gases in GaAs heterostructures [30,52]. As discussed in detail in Ref. [30], in these systems a pronounced signal due to WAL is also observed without any positive magnetoconductivity due to weak localization. The quantitative analysis of the magnetoconductivity gives an upper limit for $\tau_{\text {so }}\left(\tau_{\text {so }} \approx 3\right.$ ps in that case), such that $\tau>\tau_{\mathrm{so}}(\tau \approx 25$ ps for those systems), in complete analogy to what we find in our high-mobility graphene-on-TMD devices. For 2D holes in GaAs heterostructures, the very short $\tau_{\text {so }}$ values extracted from the analysis of WAL were taken as a signature of a strong SOI 
originating from the band structure (i.e., not from impurity scattering) [30]. This conclusion was validated through a study of $\mathrm{SdH}$ resistance oscillations that exhibit a gatevoltage-dependent beating. The beating is due to SOI that splits the hole Fermi surface, causing SdH oscillations to occur with two distinct frequencies. By analyzing the frequency splitting as a function of carrier density, the precise nature of the SOI term present in the Hamiltonian could be established.

In view of the similarity in the behavior of WAL in graphene-on-TMDs and in GaAs-based 2D hole gases, it is tempting to draw analogous conclusions. Namely, the observed behavior of WAL appears to indicate a band origin of SOI in graphene-on-TMD. Indeed, the SOIinduced splitting in the electronic band structure can generate WAL, which is at most weakly dependent on the amount of disorder in the system. This is consistent with our observation of WAL, which is strong and robust for a wide range of carrier mobilities in a variety of different samples. To confirm the band origin of interfacially induced SOI, we now proceed to search for the occurrence of a beating in the low-field $\mathrm{SdH}$ resistance oscillations.

\section{SPIN-ORBIT BAND STRUCTURE SPLITTING AND SDH OSCILLATIONS IN HIGH-MOBILITY GRAPHENE-ON-TMD DEVICES}

The SdH oscillations of transport coefficients in nonquantizing magnetic fields arise because of cyclotron motion of carrier states at the Fermi level. The periodic dependence on the inverse field $1 / B$ provides a convenient way to measure the Fermi surface size. In the presence of spin-orbital splitting, the electronic band structure gives rise to split Fermi surfaces with different spin polarization. In this regime, the $\mathrm{SdH}$ oscillations exhibit a characteristic beating pattern that provides an unambiguous diagnostic of the split Fermi surface, allowing us to directly measure the spin splitting value.

Measurement of the beating patterns in $\mathrm{SdH}$ oscillations relies on resolving a large number of Landau levels at moderate-to-low magnetic fields. Achieving this regime requires devices of exceptional quality. One constraint arises from carrier mobility, which must be high enough to prevent the washing out of Landau levels of high order. Another constraint, which is equally important, is the absence of significant inhomogeneity in carrier density across the device. Indeed, an inhomogeneous density would result in washing out of the $\mathrm{SdH}$ oscillations due to different parts of the device contributing to the $\mathrm{SdH}$ oscillations with different frequencies. If the spread in frequencies originating from the carrier inhomogeneity is comparable to (or larger than) the SOI-induced frequency splitting, no splitting can be detected experimentally.

The high-mobility values that can be achieved in our graphene-on-TMD devices are comfortably in the range needed for detecting a SOI-induced beating in the $\mathrm{SdH}$ oscillations. Charge inhomogeneity, on the contrary, poses certain challenges. Previous work indicates that, in the density range of our experiments, the inhomogeneity effects are less prominent in the graphene bilayer (BLG) as compared to the graphene monolayer [53]. This is so because the density of quasiparticle states is higher in the bilayer, where quasiparticle dispersion is quadratic, and lower in the monolayer, where the dispersion is linear [54]. We therefore employ high-mobility BLG devices for this part of the experiments.

As shown in Fig. 7(a), magnetoresistance measurements performed on high-quality BLG-on-WSe $\mathrm{W}_{2}$ indeed exhibit beating in the $\mathrm{SdH}$ oscillations. The node of the beating pattern, marked by arrows, shifts towards higher magnetic field values when a more negative gate voltage is applied. Accordingly, the $\mathrm{SdH}$ oscillation Fourier spectrum [Fig. 7(b)] exhibits a pair of peaks with a splitting that increases upon shifting $V_{g}$ further away from charge
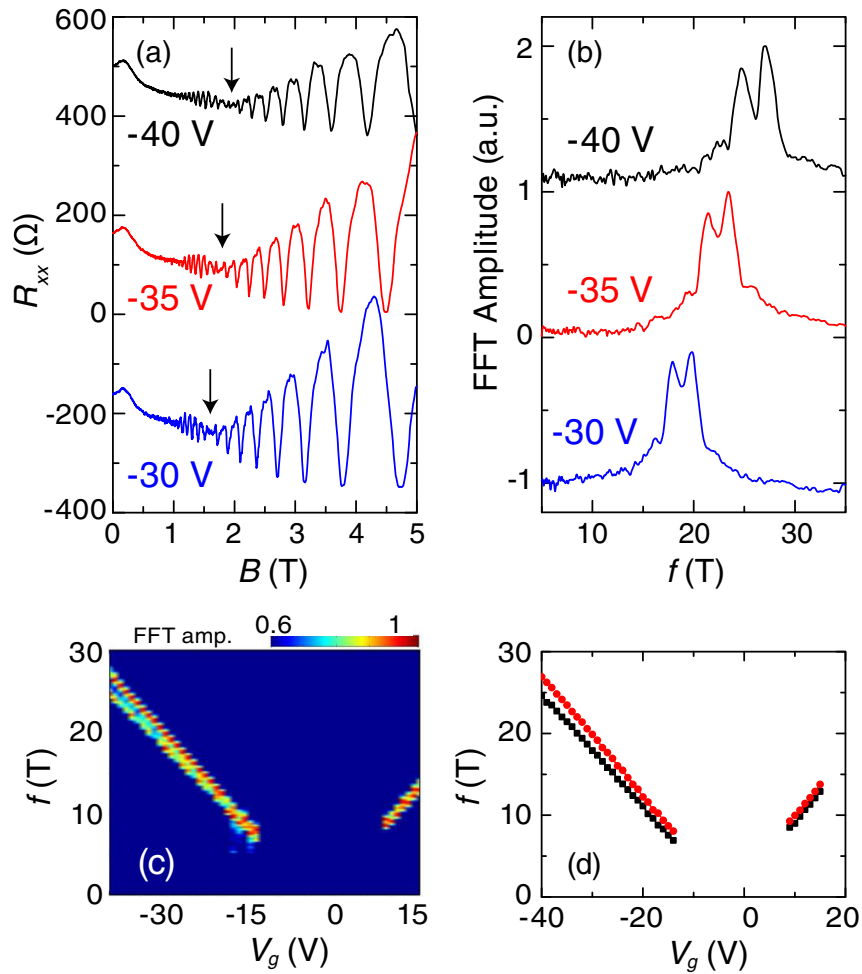

FIG. 7. Extracting SOI from the $\mathrm{SdH}$ resistance oscillations observed in BLG-on-WSe ${ }_{2}$ devices. (a) $\mathrm{SdH}$ resistance oscillations exhibit a beating pattern whose node position shifts with the applied gate voltage $V_{g}$ (curves are vertically offset for clarity; the charge neutrality point in this device is at $V_{g}=-2 \mathrm{~V}$ ). (b) Peak splitting in the Fourier spectra of the data shown in (a) is used to determine the $\mathrm{SdH}$ frequency splitting. (c) Color-coded Fourier spectrum plotted vs frequency $f$ and gate voltage $V_{g}$. (d) The position of the two peaks in the Fourier spectra shown in (c) plotted vs $V_{g}$ (black and red circles represent the lower and higher frequency peaks, respectively). The dependence on $V_{g}$ indicates that the $\mathrm{SdH}$ frequency splitting increases as the carrier density increases. 
neutrality. The full behavior is illustrated in Fig. 7(c), which shows the Fourier spectrum as a function of $V_{g}$ and oscillation frequency $f$. The frequencies corresponding to the maxima of the split peaks in the Fourier spectrum are plotted in Fig. 7(d) as a function of $V_{g}$. The observed splitting in the $\mathrm{SdH}$ oscillation frequency is a direct manifestation of the SOI splitting of the Fermi surface of BLG-on-WSe ${ }_{2}$.

These findings indicate that the effect of the interfacially induced SOI is dominated by a modification of the band structure of graphene rather than a spin-dependent disorder potential. For each value of $V_{g}$, the two peak frequencies are proportional to the areas of the two split Fermi surfaces. The measured dependence of these areas on $V_{g}$ can be used to reconstruct the band structure as described below.

However, before getting into the details of this discussion, it is useful to note that the general properties of the interfacial SOI, such as its magnitude and momentum dependence, can be inferred directly from the observed $\mathrm{SdH}$ beating patterns without invoking detailed modeling. Indeed, since for parabolic bands the Fermi energy is proportional to carrier density, the observed 10\%-15\% splitting in density translates into a $10 \%-15 \%$ splitting in the Fermi energy. This predicts the SOI splitting on the order of $10 \mathrm{meV}$ or higher. Second, the monotonic increase of the splitting vs density indicates that the SOI has a strong momentum dependence. This behavior is consistent with the dominant SOI of a Rashba type. Both conclusions are confirmed below by a detailed modeling.

The general Hamiltonian for BLG-on-TMD takes on the form of a low-energy BLG Hamiltonian with a SOI term added to describe interfacial coupling to TMD. The literature describes BLG in terms of two Dirac Hamiltonians of the two constituent monolayers coupled by the $\gamma_{1}$ term describing interlayer nearest-neighbor hopping $[47,54,55]$. The effect of interfacially induced SOI can be modeled by an effective Hamiltonian, which was determined through ab initio calculations in a previous study of monolayer graphene on $\mathrm{WS}_{2}$ [18]. The expression for the spin-orbit Hamiltonian obtained in Ref. [18] is

$$
H_{\mathrm{SOI}}=\frac{\lambda}{2} \tau_{z} s_{z} 1_{\sigma}+\frac{\lambda_{R}}{2}\left(\tau_{z} \sigma_{x} s_{y}-\sigma_{y} s_{x}\right),
$$

where Pauli matrices $s_{x, y, z}$ and $\sigma_{x, y, z}$ represent electron spin $1 / 2$ and pseudospin (sublattice A-B wave-function components), respectively. The parameters $\lambda$ and $\lambda_{R}$ represent the strengths of the two types of SOI induced by the TMD substrate, hereafter referred to as "spin-valley SOI" and Rashba SOI, respectively [18,56].

Here, we incorporate the Hamiltonian given in Eq. (2) in the part of the full BLG-on-TMD Hamiltonian describing the graphene layer in direct contact with the $\mathrm{WSe}_{2}$ substrate. We find the minimal single-valley Hamiltonian describing BLG-on-WSe $\mathrm{W}_{2}$, which reads

$$
\begin{aligned}
H= & v\left(\tau_{z} \sigma_{x} k_{x}+\sigma_{y} k_{y}\right) 1_{s} 1_{\rho}+\frac{1}{2} \gamma_{1} 1_{s}\left(\sigma_{x} \rho_{x}+\sigma_{y} \rho_{y}\right) \\
& +\frac{1}{2} U 1_{s} 1_{\sigma} \rho_{z}+\left(\frac{1_{\rho}+\rho_{z}}{2}\right) H_{\mathrm{SOI}},
\end{aligned}
$$

where $\rho_{x, y, z}$ are Pauli matrices corresponding to the BLG layer index, the quantities $\sigma_{x, y, z}$ and $s_{x, y, z}$ were defined above, the quantities $1_{\rho, \sigma, s}$ denote the corresponding $2 \times 2$ identity matrices, and $\tau_{z}= \pm 1$ is the valley index (valley degeneracy persists in the presence of SOI). The Hamiltonian $H$ includes the interlayer potential difference $U$. In the single-gated configuration of our devices, the value $U$ is finite in the presence of an applied gate voltage $V_{g}$ [55].

We use the Hamiltonian given in Eq. (3) for the quantitative estimate of the SOI parameters $\lambda$ and $\lambda_{R}$. This is done by determining the $\mathrm{SdH}$ oscillation frequency $f$ of each spin-split band from the area of the corresponding Fermi surface and by comparing the resulting values with the experimental data. To this end, we employ the approach
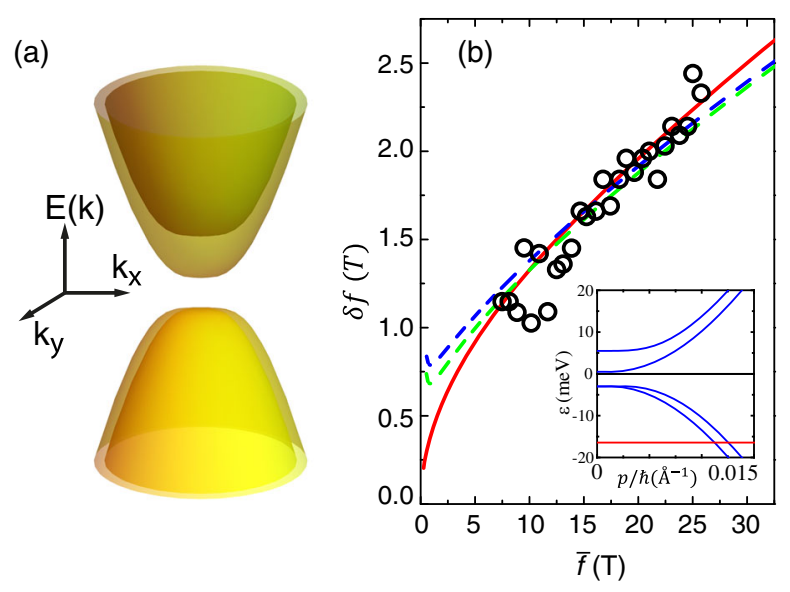

FIG. 8. (a) Low-energy band structure of bilayer graphene on TMD, obtained from the Hamiltonian in Eq. (3), with $U=-6 \mathrm{meV}, \lambda_{R}=13 \mathrm{meV}, \lambda=5 \mathrm{meV}$. See the inset of panel (b) for a numerically accurate band cross section, with the red line indicating the position of the Fermi energy calculated selfconsistently as described in the text. (b) The circles represent the measured splitting $\delta f$ of the frequency of the $\mathrm{SdH}$ resistance oscillations plotted versus the average frequency peak $\bar{f}$. The values of $\delta f$ and $\bar{f}$ are obtained from the data shown in Fig. 7(d) as the Fermi energy is swept through the valence band $\left(V_{g}<-2 \mathrm{~V}\right)$. The colored lines correspond to theoretical predictions calculated using the band structure obtained with the Hamiltonian in Eq. (3) for different values of $\lambda_{R}$ and $\lambda$. Three possible best-fit plots with $\left(\lambda_{R}, \lambda\right)=(15,0) \mathrm{meV}$ (red solid line), $\left(\lambda_{R}, \lambda\right)=(13,-5) \mathrm{meV}$ (green, dashed line), and $\left(\lambda_{R}, \lambda\right)=$ $(13,5) \mathrm{meV}$ (blue, dashed-dotted line) are shown. Note the electron-hole asymmetry in the band structure and, in particular, in the spin splitting, clearly visible in the inset of panel (b). As discussed in the main text, such an asymmetry may allow full gate tuning of SOI in double-gated bilayer devices. 
of Ref. [57] for obtaining $E_{F}$ self-consistently. For a given $\lambda, \lambda_{R}$, and $U$, the band structure can be easily computed; see Fig. 8(a) for an example. Because of the layer asymmetry, eigenstates of the Hamiltonian Eq. (3) are not equally partitioned between the two graphene layers of the BLG. Thus, just as in the case of the spin-degenerate gapped BLG, the total carrier density $n=n_{1}+n_{2}$ fixed by a given $E_{F}$ is unequally split between the two layers. The unequal layer carrier densities give rise to an interlayer potential difference $\tilde{U}(n)$ that depends on the total carrier density $n$ [58]. By varying $E_{F}$ (or $n$ ), we can find the self-consistent value for which $\tilde{U}(n)=U$. The self-consistent solution corresponds to the experimental situation in which the gate voltage $V_{g}$ is given by $C_{\square}\left(V_{g}-V_{g 0}\right)=e n$, where $V_{g 0}$ corresponds to the charge neutrality point and $C_{\square}$ is the capacitance per unit area between the device and gate electrode. As an illustration, the red line in the inset of Fig. 8(b) indicates the position of the self-consistent value of $E_{F}$ for $U=-6 \mathrm{meV}, \lambda_{R}=13 \mathrm{meV}$, and $\lambda=5 \mathrm{meV}$.

Knowing the self-consistent $E_{F}$ for a given $U$, we can then determine the Fermi momenta $k_{F+}$ and $k_{F-}$ of the two spin-split bands, or-equivalently - the areas of the corresponding Fermi surfaces and the density of carriers $n_{+}$and $n_{-}$in these two bands (note that $n=n_{+}+n_{-}$). For instance, in the inset of Fig. 8(b), $k_{F+}$ and $k_{F-}$ correspond to the intersection of the red line with the two blue lines. This procedure is then repeated so that we obtain selfconsistent solutions of $n_{+}$and $n_{-}$as $U$ (and hence $E_{F}$ ) is swept through the valence band, just as is done in experiments. The values of the frequency peaks are related to the carrier densities through rescaling by a factor of $h / 2 e$.

Having self-consistently determined the relation between $n_{ \pm}$and $U$, we proceed to compare theory with experimental results. This is done by plotting the frequency splitting $\delta f$ as a function of the average frequency $\bar{f}$. Here, both quantities can be extracted directly and independently from the measured data in Fig. 7(d) (note that $\bar{f} \propto V_{g}$ ). In Fig. 8(b), the empty circles correspond to the experimental data measured as $E_{F}$ is swept through the valence band, while the colored lines represent three possible theoretical best-fit plots obtained for different combinations of SOI values $\left(\lambda_{R}, \lambda\right)=(15,0) \mathrm{meV}$ (red solid line), $\left(\lambda_{R}, \lambda\right)=$ $(13,-5) \mathrm{meV}$ (green, dashed line), and $\left(\lambda_{R}, \lambda\right)=(13,5)$ (blue, dashed-dotted line). In this way, a very satisfactory fit to the data can be obtained. Upon varying $\lambda_{R}$ and $\lambda$ over a broad interval, we find that the range of values for which a good agreement is found is $\lambda_{R} \simeq 10-15 \mathrm{meV}$ and $\lambda \simeq 0-6 \mathrm{meV}$ (larger values of $\lambda_{R}$ constrain $\lambda$ to smaller values to fit the data).

Theory can also reproduce the data obtained when $E_{F}$ is swept through the conduction band, but in that case, the range of carrier density for which a beating is observed experimentally is smaller [see Fig. 7(d)], and thus only a few data points are present in the $\delta f$-vs- $\bar{f}$ plot (again, $\bar{f} \propto V_{g}$ ). In the conduction band, the best-fit values of $\lambda_{R}$ and $\lambda$ are in the range between 5 and $8 \mathrm{meV}$. Although in this case SOI appears to be slightly weaker than in the valence band, the smaller amount of data makes it more difficult to determine the two parameters accurately.

Our analysis of the beating patterns observed in the $\mathrm{SdH}$ measurements therefore indicates that the strong SOI induced in graphene by proximity with TMD is of a band origin. The SOI strength extracted from the comparison between theory and experiments is in the same ballpark, although somewhat larger than the values estimated in Ref. [18] from $a b$ initio calculations. The similarity of the SOI strength estimated from the data and that found from $a b$ initio calculations supports the consistency of our analysis. We therefore conclude that the characteristic magnitude of the interfacially induced SOI in graphene is about $10 \mathrm{meV}$. This value is more than 100 times larger than the SOI intrinsically present in pristine graphene [22-24].

This result is also in line with the conclusion drawn from the behavior of the spin-relaxation time $\tau_{\mathrm{so}}$ obtained from the analysis of WAL. In this regard, we note that a nearly 10-meV spin splitting in the band structure, such as the one we found, would give rise to a characteristic "Rabi" spinprecession frequency of $\lambda_{R} / \hbar \approx 10 \mathrm{THz}$, which is compatible with values of the spin relaxation time $\tau_{\mathrm{so}}^{*} \approx 0.2 \mathrm{ps}$ inferred from WAL. Indeed, it would have been difficult to reconcile this fast spin relaxation time with a much smaller SOI strength - say, a spin splitting of $1 \mathrm{meV}$ or less. Specifically, a 1-meV spin splitting corresponds to a $1-\mathrm{THz}$ Rabi frequency, which is insufficient to randomize the spin direction over a time of $0.1-0.2$ ps. These considerations indicate that two very different phenomena-the quantum correction to the conductivity due to electron interference and the splitting in the frequency of the $\mathrm{SdH}$ oscillations-lead to conclusions on the estimated strength of the SOI that are internally consistent, providing an independent confirmation of the validity of our analysis.

\section{CONCLUSION}

The main conclusion that can be drawn from the measurements presented above is that the interfacially induced SOI is dominated by spin-orbital splitting in the graphene band structure. This generalizes to spindependent phenomena the results obtained in grapheneon-hBN moire superlattices, where interfacial interactions alter the graphene band structure by producing secondary Dirac points and creating a gap at the main Dirac point. Our second conclusion is that the interfacially induced SOI is extremely robust. A strong SOI is induced irrespective of the specific TMD material used, of the graphene and TMD lattice alignment angle, of the thickness of the graphene multilayer (which we tested up to three layers), or of the position of the Fermi level in the graphene band. 
Most tellingly, we observe a pronounced magnetoconductivity due to weak antilocalization in all devices that we have measured at low temperatures, where the transport regime varied from fully diffusive up to nearly ballistic. None of the devices exhibited a positive magnetoconductivity due to weak localization. This indicates that SOI in graphene was-in all regimes investigated-sufficiently strong to cause a full precession of the electron spin even for the shortest trajectories that contribute to electron interference. These observations appear to only be compatible with a band origin of strong SOI, a conclusion that is confirmed by the experimental observation of beating patterns and a splitting in the frequency of the Shubnikovde Haas oscillations in high-quality bilayer devices. The evolution in the magnitude of the splitting that is observed upon varying carrier density indicates that the dominant contribution to the interfacially induced SOI is of the Rashba type. A quantitative analysis of the data indicates that the interaction coupling constant for the Rashba term is as large as $10-15 \mathrm{meV}$, whereas the strength of the other SOI term that couples spin and valley degrees of freedom is about 5-6 meV or smaller. These values correspond to a remarkably strong SOI, especially in comparison to the minute values of the intrinsic SOI in graphene, which are only 20-40 $\mu \mathrm{eV}$ [22-24].

It is remarkable that such a large interfacial SOI can be induced without causing any damage to the electronic properties of graphene. Our highest quality devices exhibited carrier mobility reaching up to $160000 \mathrm{~cm}^{2} / \mathrm{Vs}$ and carrier density inhomogeneity of only $\simeq 2 \times 10^{10} \mathrm{~cm}^{-2}$, which is comparable to the best graphene-on-hBN devices $[2-4,39,40]$. The possibility to achieve such a high quality, both in terms of carrier mobility and density homogeneity, will be crucial for probing the predicted topologically insulating states that may be realized in graphene-based systems $[18,25,26]$.

In that regard, we also note that, since Rashba turns out to be the dominant SOI coupling, a gap opening between valence and conduction bands in charge-neutral grapheneon-TMD (leading to a topologically insulating state) is not expected to occur [18]. However, diverse strategies are available to change the situation. For instance, encapsulating graphene in between two TMD crystals may result in a smaller asymmetry of the device structure, causing a decrease in the intensity of the Rashba term, with other spin-valley SOI contributions becoming stronger. Under these conditions, a topological insulating state may be engineered in graphene with a band gap of several meV [18] and, if so, the ability to achieve very high carrier mobility and small density inhomogeneity demonstrated in this work will be essential for probing the occurrence of edge transport in the presence of an insulating bulk.

A new opportunity opened up by the results presented here is achieving gate control of SOI in graphene-based systems. A simple strategy is to employ dual-gated BLG devices, using a TMD layer as a gate insulator on one side and a hBN layer on the other side. The application of a perpendicular electric field in such a structure will lead to a band-gap opening at the charge neutrality point [31-33]. In this regime, electronic states at the top of the valence band and at the bottom of the conduction band have their wave functions localized on one of the two BLG layers, depending on the sign of the perpendicular electric field [59]. Since the interfacially induced SOI is present mostly in the BLG layer in direct contact with the TMD, the spin splitting will occur predominantly in the band whose states are localized in that layer. As a result, for a fixed perpendicular electric field, the band structure will become electron-hole asymmetric. Interestingly, the presence of a pronounced electron-hole asymmetry is evident in the inset of Fig. 8(b). The "on" and "off" switching of SOI is then enabled by a dualgated configuration, which allows the position of the Fermi level to be tuned without changing the polarity and magnitude of the electric field responsible for the gap opening. In this scheme, the SOI intensity can be fully turned on and off, at least in principle, because at the conduction and valence band edges, the wave function is $100 \%$ layer polarized. However, the observation of this spin-induced electron-hole asymmetry is challenging in the single-gated devices investigated here because varying the gate voltage to change the position of the Fermi level from the valence to the conduction band also results in reversing the polarity of the applied perpendicular electric field.

Finally, strong interfacially induced SOI in graphene-onTMDs creates countless opportunities for investigating novel physical phenomena under controlled conditions. For instance, devices can be realized by employing ferromagnetic electrical contacts that enable injection and detection of spins in graphene [20,60]. In these systems, the dynamics of spin-polarized carriers will be controlled, and altered in an interesting way, by the interfacially induced SOI. These systems will also help us to gain new insight into the subtle phenomena originating from the spin-Hall effect and the inverse spin-Hall effect [61]. These examples illustrate that the ability to engineer the properties of electronic systems in van der Waals heterostructures through a layer-by-layer assembly-demonstrated here for the case of SOI in graphene-opens up a wide range of exciting opportunities for realizing and exploring new physical phenomena.

\section{ACKNOWLEDGMENTS}

We gratefully acknowledge technical assistance from A. Ferreira. Z. W., D. K., D. M., and A. F. M. also gratefully acknowledge financial support from the Swiss National Science Foundation, The National Centre of Competence in Research 'Quantum Science and Technology', and the EU Graphene Flagship Project. J. K. is supported by the National Science Scholarship from the Agency for Science, Technology and Research (A*STAR). 


\section{APPENDIX: CLASSICAL BACKGROUND IN THE MAGNETOCONDUCTANCE}

As discussed in Sec. V, for the quantitative analysis of the WAL data, the quantum correction to the magnetoconductivity is obtained by subtracting the classical contribution from the total measured magnetoconductivity. The classical contribution is determined by taking the magnetoconductivity measured at a temperature that is sufficiently high to significantly reduce the phase coherence time $\tau_{\phi}$. In the case of our devices, this happens at 20-30 K, above which quantum corrections become negligible (as indicated, for instance, by the complete disappearance of the negative magnetoconductivity due to WAL).

Here, we briefly discuss the behavior of the classical contribution that we subtract, which is shown in Fig. 9 for three devices with different mobility and mean-free path $(l)$. It is seen that the classical magnetoconductivity is small and negative for the low-mobility device (green line in Fig. 9, $\mu \simeq 3000 \mathrm{~cm}^{2} / \mathrm{Vs}$ ) as expected from diffusive Drude transport. For these low-mobility devices, the background is so small that it has virtually no effect on the quantitative analysis of the data; i.e., removing it or not leads to virtually identical estimates of $\tau_{s o}$. For devices with larger mobility, we systematically find that the classical magnetoconductivity tends to become positive, growing in magnitude with increasing mean-free path. This behavior can be understood by recalling that, while the classical magnetoconductivity is negative in the diffusive regime, it changes sign and becomes positive in a narrow channel in which the mean-free path is larger than the channel width [62]. In the crossover regime, occurring when the mean-free path increases and approaches the channel width, the magnetoconductivity is positive but small, growing larger for larger mean-free path values. Although the devices that we have studied are never fully in this regime, in some cases, the mean-free path is close to half of the channel width (black line in Fig. 9).

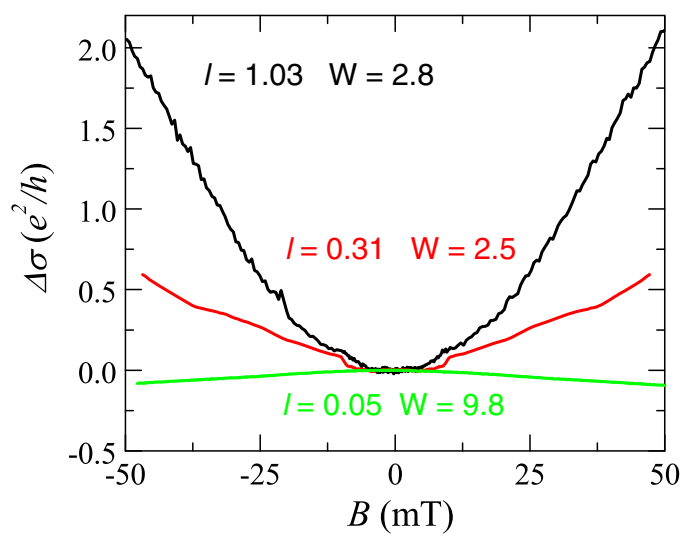

FIG. 9. Magnetoconductivity measured at high temperatures (20-30 K depending on the device) in the devices with different mean-free paths $(l$, in $\mu \mathrm{m})$. The width $(W$, in $\mu \mathrm{m})$ of the device is also indicated.
We mention this point because it might appear that the positive-background magnetoconductivity that we subtract is in fact due to weak localization. The data, however, show that this is not the case. Not only is there an absence of temperature dependence in the background [see Figs. 2(a)-2(c)], but also the magnitude of the measured positive magnetoconductivity typically far exceeds $e^{2} / h$ (see, for instance, the black curve in Fig. 9). Both features are incompatible with the weak-localization interpretation. Additionally, in highmobility devices with a long mean-free path, the weaklocalization signal should appear only at low magnetic fields (within a few $\mathrm{mT}$ ) when the magnetic length is longer than (or, at most, comparable to) the mean-free path, whereas the observed positive magnetoconductivity continues to increase up to much higher magnetic field values.

[1] A. K. Geim and I. V. Grigorieva, Van der Waals Heterostructures, Nature (London) 499, 419 (2013).

[2] C. R. Dean et al., Hofstadter's Butterfly and the Fractal Quantum Hall Effect in Moire Superlattices, Nature (London) 497, 598 (2013).

[3] L. A. Ponomarenko et al., Cloning of Dirac Fermions in Graphene Superlattices, Nature (London) 497, 594 (2013).

[4] B. Hunt, J. D. Sanchez-Yamagishi, A. F. Young, M. Yankowitz, B. J. LeRoy, K. Watanabe, T. Taniguchi, P. Moon, M. Koshino, P. Jarillo-Herrero, and R. C. Ashoori, Massive Dirac Fermions and Hofstadter Butterfly in a van der Waals Heterostructure, Science 340, 1427 (2013).

[5] W. J. Yu, Y. Liu, H. Zhou, A. Yin, Z. Li, Y. Huang, and X. Duan, Highly Efficient Gate-Tunable Photocurrent Generation in Vertical Heterostructures of Layered Materials, Nat. Nanotechnol. 8, 952 (2013).

[6] L. Britnell, R. M. Ribeiro, A. Eckmann, R. Jalil, B. D. Belle, A. Mishchenko, Y.-J. Kim, R. V. Gorbachev, T. Georgiou, S. V. Morozov et al., Strong Light-Matter Interactions in Heterostructures of Atomically Thin Films, Science 340, 1311 (2013).

[7] C.-H. Lee et al., Atomically Thin p-n Junctions with van der Waals Heterointerfaces, Nat. Nanotechnol. 9, 676 (2014).

[8] Y.-M. Li, J. Li, L.-K. Shi, D. Zhang, W. Yang, and K. Chang, Light-Induced Exciton Spin Hall Effect in van der Waals Heterostructures, Phys. Rev. Lett. 115, 166804 (2015).

[9] P. Rivera et al., Observation of Long-Lived Interlayer Excitons in Monolayer $\mathrm{MoSe}_{2}-\mathrm{WSe}_{2}$ Heterostructures, Nat. Commun. 6, 6242 (2015).

[10] Z. Wang, C. Tang, R. Sachs, Y. Barlas, and J. Shi, Proximity-Induced Ferromagnetism in Graphene Revealed by the Anomalous Hall Effect, Phys. Rev. Lett. 114, 016603 (2015).

[11] M. Gmitra and J. Fabian, Graphene on Transition-Metal Dichalcogenides: A Platform for Proximity Spin-Orbit Physics and Optospintronics, Phys. Rev. B 92, 155403 (2015).

[12] Z. Y. Zhu, Y.C. Cheng, and U. Schwingenschlögl, Giant Spin-Orbit-Induced Spin Splitting in Two-Dimensional Transition-Metal Dichalcogenide Semiconductors, Phys. Rev. B 84, 153402 (2011). 
[13] D. Xiao, G.-B. Liu, W. Feng, X. Xu, and W. Yao, Coupled Spin and Valley Physics in Monolayers of $\mathrm{MoS}_{2}$ and Other Group-VI Dichalcogenides, Phys. Rev. Lett. 108, 196802 (2012).

[14] K. Kośmider, J. W. González, and J. Fernández-Rossier, Large Spin Splitting in the Conduction Band of Transition Metal Dichalcogenide Monolayers, Phys. Rev. B 88, 245436 (2013).

[15] Q. H. Wang, K. Kalantar-Zadeh, A. Kis, J. N. Coleman, and M.S. Strano, Electronics and Optoelectronics of Two-Dimensional Transition Metal Dichalcogenides, Nat. Nanotechnol. 7, 699 (2012).

[16] A. V. Kretinin et al., Electronic Properties of Graphene Encapsulated with Different Two-Dimensional Atomic Crystals, Nano Lett. 14, 3270 (2014).

[17] A. Avsar et al., Spin-Orbit Proximity Effect in Graphene, Nat. Commun. 5, 4875 (2014).

[18] Z. Wang, D.-K. Ki, H. Chen, H. Berger, A. H. MacDonald, and A. F. Morpurgo, Strong Interface-Induced Spin-Orbit Interaction in Graphene on $\mathrm{WS}_{2}$, Nat. Commun. 6, 8339 (2015).

[19] B. Yang, M.-F. Tu, J. Kim, Y. Wu, H. Wang, J. Alicea, R. Wu, M. Bockrath, and J. Shi, Tunable spin-orbit coupling and symmetry-protected edge states in graphene/ $\mathrm{WS}_{2}$, 2D Mater. 3, 031012 (2016).

[20] N. Tombros, C. Jozsa, M. Popinciuc, H. T. Jonkman, and B. J. van Wees, Electronic Spin Transport and Spin Precession in Single Graphene Layers at Room Temperature, Nature (London) 448, 571 (2007).

[21] M. H. D. Guimarães, P. J. Zomer, J. Ingla-Aynés, J. C. Brant, N. Tombros, and B. J. van Wees, Controlling Spin Relaxation in Hexagonal BN-Encapsulated Graphene with a Transverse Electric Field, Phys. Rev. Lett. 113, 086602 (2014).

[22] D. Huertas-Hernando, F. Guinea, and A. Brataas, Spin-Orbit Coupling in Curved Graphene, Fullerenes, Nanotubes, and Nanotube Caps, Phys. Rev. B 74, 155426 (2006).

[23] H. Min, J. E. Hill, N. A. Sinitsyn, B. R. Sahu, L. Kleinman, and A.H. MacDonald, Intrinsic and Rashba Spin-Orbit Interactions in Graphene Sheets, Phys. Rev. B 74, 165310 (2006).

[24] S. Konschuh, M. Gmitra, and J. Fabian, Tight-Binding Theory of the Spin-Orbit Coupling in Graphene, Phys. Rev. B 82, 245412 (2010).

[25] C. L. Kane and E. J. Mele, $Z_{2}$ Topological Order and the Quantum Spin Hall Effect, Phys. Rev. Lett. 95, 146802 (2005).

[26] C. L. Kane and E. J. Mele, Quantum Spin Hall Effect in Graphene, Phys. Rev. Lett. 95, 226801 (2005).

[27] M. Gmitra, D. Kochan, P. Högl, and J. Fabian, Trivial and Inverted Dirac Bands and the Emergence of Quantum Spin Hall States in Graphene on Transition-Metal Dichalcogenides, Phys. Rev. B 93, 155104 (2016).

[28] B. Das, D. C. Miller, S. Datta, R. Reifenberger, W. P. Hong, P. K. Bhattacharya, J. Singh, and M. Jaffe, Evidence for Spin Splitting in $\mathrm{In}_{x} \mathrm{Ga}_{1-x} \mathrm{As} / \mathrm{In}_{0.52} \mathrm{Al}_{0.48}$ As Heterostructures as B $\rightarrow$ 0, Phys. Rev. B 39, 1411 (1989).

[29] J. Nitta, T. Akazaki, H. Takayanagi, and T. Enoki, Gate Control of Spin-Orbit Interaction in an Inverted $\mathrm{In}_{0.53} \mathrm{Ga}_{0.47} \mathrm{As} / \mathrm{In}_{0.52} \mathrm{Al}_{0.48}$ As Heterostructure, Phys. Rev. Lett. 78, 1335 (1997).
[30] B. Grbić, R. Leturcq, T. Ihn, K. Ensslin, D. Reuter, and A. D. Wieck, Strong Spin-Orbit Interactions and Weak Antilocalization in Carbon-Doped p-type $\mathrm{GaAs} / \mathrm{Al}_{x} \mathrm{Ga}_{1-x} \mathrm{As}$ Heterostructures, Phys. Rev. B 77, 125312 (2008).

[31] J. B. Oostinga, H. B. Heersche, X. Liu, A. F. Morpurgo, and L. M. K. Vandersypen, Gate-Induced Insulating State in Bilayer Graphene Devices, Nat. Mater. 7, 151 (2008).

[32] R. T. Weitz, M. T. Allen, B. E. Feldman, J. Martin, and A. Yacoby, Broken-Symmetry States in Doubly Gated Suspended Bilayer Graphene, Science 330, 812 (2010).

[33] J. Velasco et al., Transport Spectroscopy of SymmetryBroken Insulating States in Bilayer Graphene, Nat. Nanotechnol. 7, 156 (2012).

[34] M. F. Craciun, S. Russo, M. Yamamoto, J. B. Oostinga, A. F. Morpurgo, and S. Tarucha, Trilayer Graphene Is a Semimetal with a Gate-Tunable Band Overlap, Nat. Nanotechnol. 4, 383 (2009).

[35] C. R. Dean, A. F. Young, I. Meric, C. Lee, L. Wang, S. Sorgenfrei, K. Watanabe, T. Taniguchi, P. Kim, K. L. Shepard, and J. Hone, Boron Nitride Substrates for HighQuality Graphene Electronics, Nat. Nanotechnol. 5, 722 (2010).

[36] N. J. G. Couto, D. Costanzo, S. Engels, D.-K. Ki, K. Watanabe, T. Taniguchi, C. Stampfer, F. Guinea, and A. F. Morpurgo, Random Strain Fluctuations as Dominant Disorder Source for High-Quality On-Substrate Graphene Devices, Phys. Rev. X 4, 041019 (2014).

[37] K. Kim, S. Larentis, B. Fallahazad, K. Lee, J. Xue, D. C. Dillen, C. M. Corbet, and E. Tutuc, Band Alignment in $\mathrm{WSe}_{2}$-Graphene Heterostructures, ACS Nano, 9, 4527 (2015).

[38] A. S. Mayorov, R. V. Gorbachev, S. V. Morozov, L. Britnell, R. Jalil, L. A. Ponomarenko, P. Blake, K. S. Novoselov, K. Watanabe, T. Taniguchi, and A. K. Geim, Micrometer-Scale Ballistic Transport in Encapsulated Graphene at Room Temperature, Nano Lett. 11, 2396 (2011).

[39] A. F. Young, C. R. Dean, L. Wang, H. Ren, P. CaddenZimansky, K. Watanabe, T. Taniguchi, J. Hone, K. L. Shepard, and P. Kim, Spin and Valley Quantum Hall Ferromagnetism in Graphene, Nat. Phys. 8, 550 (2012).

[40] T. Taychatanapat, K. Watanabe, T. Taniguchi, and P. JarilloHerrero, Electrically Tunable Transverse Magnetic Focusing in Graphene, Nat. Phys. 9, 225 (2013).

[41] A. M. Goossens, V. E. Calado, A. Barreiro, K. Watanabe, T. Taniguchi, and L. M. K. Vandersypen, Mechanical Cleaning of Graphene, Appl. Phys. Lett. 100, 073110 (2012).

[42] S. Hikami, A. I. Larkin, and Y. Nagaoka, Spin-Orbit Interaction and Magnetoresistance in the Two Dimensional Random System, Theor. Math. Phys. 63, 707 (1980).

[43] C. W. J. Beenakker and H. van Houten, Quantum Transport in Semiconductor Nanostructures, Solid State Phys. 44, 1 (1991).

[44] P. A. Lee and A. Douglas Stone, Universal Conductance Fluctuations in Metals, Phys. Rev. Lett. 55, 1622 (1985).

[45] G. Bergmann, Physical Interpretation of Weak Localization: A Time-of-Flight Experiment with Conduction Electrons, Phys. Rev. B 28, 2914 (1983).

[46] K. S. Novoselov, E. McCann, S. V. Morozov, V. I. Fal'ko, M. I. Katsnelson, U. Zeitler, D. Jiang, F. Schedin, and 
A. K. Geim, Unconventional Quantum Hall Effect and Berry's Phase of $2 \pi$ in Bilayer Graphene, Nat. Phys. 2, 177 (2006).

[47] E. McCann and V. I. Fal'ko, Landau-Level Degeneracy and Quantum Hall Effect in a Graphite Bilayer, Phys. Rev. Lett. 96, 086805 (2006).

[48] F. V. Tikhonenko, A. A. Kozikov, A. K. Savchenko, and R. V. Gorbachev, Transition Between Electron Localization and Antilocalization in Graphene, Phys. Rev. Lett. 103, 226801 (2009).

[49] R. V. Gorbachev, F. V. Tikhonenko, A. S. Mayorov, D. W. Horsell, and A. K. Savchenko, Weak Localization in Bilayer Graphene, Phys. Rev. Lett. 98, 176805 (2007).

[50] E. McCann and V.I. Fal'ko, $z \rightarrow-z$ Symmetry of Spin-Orbit Coupling and Weak Localization in Graphene, Phys. Rev. Lett. 108, 166606 (2012).

[51] When fitting the data from different devices, we find thatin agreement with the assumptions made by theory-the condition $\tau_{\phi}>\tau_{\text {asy }}>\tau_{\text {so }}$ is always satisfied. However, whereas the data analysis gives an upper bound for $\tau_{\text {so }} \simeq$ $0.2-0.4 \mathrm{ps}$ for all devices, the spread in the values extracted for $\tau_{\text {asy }}$ is larger. For different devices, we find that $\tau_{\text {asy }}$ varies between 2 and 10 ps. Such a spread is likely to be caused-at least in part-by uncertainties in extracting the magnetoconductivity $(\Delta \sigma)$ from the measured magnetoresistance due to the limited precision in determining the device geometry (indeed, the value of $\tau_{\text {asy }}$ extracted from the fit is particularly sensitive to the absolute magnitude of the negative magnetoconductivity).

[52] J. B. Miller, D. M. Zumbühl, C. M. Marcus, Y. B. LyandaGeller, D. Goldhaber-Gordon, K. Campman, and A. C. Gossard, Gate-Controlled Spin-Orbit Quantum Interference Effects in Lateral Transport, Phys. Rev. Lett. 90, 076807 (2003).

[53] D. K. Efetov, L. Wang, C. Handschin, K. B. Efetov, J. Shuang, R. Cava, T. Taniguchi, K. Watanabe, J. Hone, C. R. Dean, and P. Kim, Specular Interband Andreev Reflections at van der Waals Interfaces between Graphene and $\mathrm{NbSe}_{2}$, Nat. Phys. 12, 328 (2016).
[54] A. H. Castro Neto, F. Guinea, N. M. R. Peres, K. S. Novoselov, and A. K. Geim, The Electronic Properties of Graphene, Rev. Mod. Phys. 81, 109 (2009).

[55] E. McCann and M. Koshino, The Electronic Properties of Bilayer Graphene, Rep. Prog. Phys. 76, 056503 (2013).

[56] We note, parenthetically, that in Ref. [18], the Hamiltonian in Eq. (2) was discussed as part of a larger Hamiltonian that contained a potential term describing sublattice A-B modulation, $H^{\prime}=(\Delta / 2) \sigma_{z} 1_{s}$. Under realistic experimental conditions, the value $\Delta$ was estimated to be very small, which makes the term $H^{\prime}$ entirely negligible.

[57] E. McCann, Asymmetry Gap in the Electronic Band Structure of Bilayer Graphene, Phys. Rev. B 74, 161403 (2006).

[58] More specifically, for a given $E_{F}$, we compute the total excess (i.e., relative to the background density of positive charges) electron density on each layer, including those from the completely filled bands. Because of the layer asymmetry of the system, eigenstates from the completely filled bands are also partitioned unequally between the two layers and thus also contribute (albeit independent of $E_{F}$ ) to the interlayer potential $U_{n} . U_{n}$ is then given by $C_{\mathrm{BLG}} U_{n}=e \delta n_{\text {total }, 2}$, where $C_{\mathrm{BLG}}$ is the capacitance per unit area of the bilayer graphene (assuming vacuum dielectric constant $\epsilon_{0}$ ) and $\delta n_{\text {total, } 2}$ is the total excess electron density on the layer further away from the substrate.

[59] A. F. Young and L. S. Levitov, Capacitance of Graphene Bilayer as a Probe of Layer-Specific Properties, Phys. Rev. B 84, 085441 (2011).

[60] W. Han, R. K. Kawakami, M. Gmitra, and J. Fabian, Graphene Spintronics, Nat. Nanotechnol. 9, 794 (2014).

[61] T. Jungwirth, J. Wunderlich, and K. Olejnik, Spin Hall Effect Devices, Nat. Mater. 11, 382 (2012).

[62] H. van Houten, C. W. J. Beenakker, P. H.M. van Loosdrecht, T. J. Thornton, H. Ahmed, M. Pepper, C. T. Foxon, and J. J. Harris, Four-Terminal Magnetoresistance of a Two-Dimensional Electron-Gas Constriction in the Ballistic Regime, Phys. Rev. B 37, 8534 (1988). 